\title{
Transverse Vibration of Circular Double-Layer Graphene Sheets Using Nonlocal Elasticity Theory
}

Haw-Long Lee, Yu-Ching Yang and Win-Jin Chang

Department of Mechanical Engineering, Kun Shan University, No.195, Kunda Rd., Yongkang Dist., Tainan

71003, Taiwan

Graphene possesses excellent physical and chemical properties and has potential applications in nanobiological devices and nanoelectromechanical systems such as actuators [1], optoelectronics [2], and biosensors [3]. In addition, the vibrational properties play an important role in structural stability of nanoelectromechanical systems used in dynamic environments. In the recent years, several researchers investigated the vibration behavior of graphene sheets using continuum models [4-5]. In order to avoid overestimating vibrational responses, it is necessary to consider the small scale effect in the analysis of nanostructures [6]. In this article, free transverse vibration of a circular double-layer graphene is studied based on nonlocal elasticity theory.

The circular double-layer graphene sheets with the radius $R$ is depicted in Fig. 1. The vibration equation for the nonlocal constitutive relations of the graphene is given by

$$
\begin{aligned}
& D \nabla^{4} w_{1}+\left[1-\left(e a_{0}\right)^{2} \nabla^{2}\right]\left[\rho h\left(\partial^{2} w_{1} / \partial t^{2}\right)+k\left(w_{1}-w_{2}\right)\right]=0 \\
& D \nabla^{4} w_{2}+\left[1-\left(e a_{0}\right)^{2} \nabla^{2}\right]\left[\rho h\left(\partial^{2} w_{2} / \partial t^{2}\right)+k\left(w_{2}-w_{1}\right)\right]=0
\end{aligned}
$$

where $w_{1}$ and $w_{2}$ are the displacement along the thickness $h$ of the graphene; $\mathrm{p}$ is the mass density; $D$ is its flexural bending rigidity which is expressed as $D=E h^{3} / 12\left(1-\mu^{2}\right)$ in which $E$ and $\mu$ are Young's modulus and Poisson's ratio, respectively; $r$ and $\theta$ are the radial and circumferential coordinates; $\nabla^{2}=\partial^{2} / d r^{2}+(1 / r) \partial / \partial r+\left(1 / r^{2}\right) \partial^{2}$ $/ \partial \theta^{2}$ is the Laplacian operator in polar coordinates. $e_{0} a$ is the nonlocal parameter revealing the nanoscale size effect; $k$ is a linear spring constant which is used to simulate the van der Waals force (i.e., confined force) between the upper and lower layers.

The corresponding clamped boundary conditions are

$$
w_{j}(R)=0, \quad \partial w_{j}(R) / \partial r=0, j=1,2
$$

In order to solve Eqs. (1) and (2), we define $f_{1}=w_{1}+w_{2}$ and $f_{2}=w_{1}-w_{2}$ which are the in-phase and antiphase solutions, respectively.

The in-phase and anti-phase solutions can be expressed as follows:

$f_{1}(r, \theta, t)=e^{i \omega t} g_{1}(r) \cos n \theta, \quad f_{2}(r, \theta, t)=e^{i \omega t} g_{2}(r) \cos n \theta$

where $\omega$ is the angular frequency of vibration and $n$ is the number of nodal diameters. Substituting Eq. (4) into Eqs. (1) and (2) and using the boundary conditions (3), we can obtained the in-phase and anti- phase frequency characteristic equation $\mathrm{F}\left(\mathrm{m}, \mathrm{n}, \omega_{\mathrm{mn}}\right)=0$, in which $m$ is the mode number.

The following material properties and geometrical parameters of graphene are used [7]: $E=1 \mathrm{TPa} ; \mu=0.3 ; h=$ $0.34 \mathrm{~nm} ; \rho=2300 \mathrm{~kg} / \mathrm{m}^{3} ; k=-108 \mathrm{GPa} / \mathrm{nm}$. Fig. 2 shows the in-phase and anti-phase frequency of the graphene sheets with $n=0$ for different mode numbers. The van der Waals force between two layers is significant for vibration behaviors of the graphene sheets. It makes a stiffer structure and results in a higher vibration frequency. This indicates that the effect of the van der Waals force on the anti-phase frequency is apparently higher than that on the in-phase frequency. In addition, the stiffness of the graphene sheets decreases when the nonlocal parameter is considered. Therefore, the in-phase and anti-phase frequencies in this study are more than those obtained by Natuski et al. [7]. It can be seen that the nonlocal effect on the frequency of the graphene sheets is more significant for the higher modes.

References:

[1]S. Park, et al, Small 6( 2010) p.210.

[2]P. Avouris and C. Dimitrakopoulos, Mater. Today 15 (2012) p.86.

[3]H. Bao, Y. Pan, and L. Li, Nano Life 2 (2012) p.1230001.

[4]R. Chowdhury et al, J. Phys. D: Appl. Phys. 44 (2011) p.205401. 
[5]J. Wang, M. Tian, and X. He, Procedia Eng. 31 (2012) p.641.

[6]A. C. Eringen, Int. J. Eng. Sci. 10 (1972) p.425.

[7]T. Natsuki, J.X. Shi, and Q.Q. Ni, J. Appl. Phys. 111 (2012) p.044310.

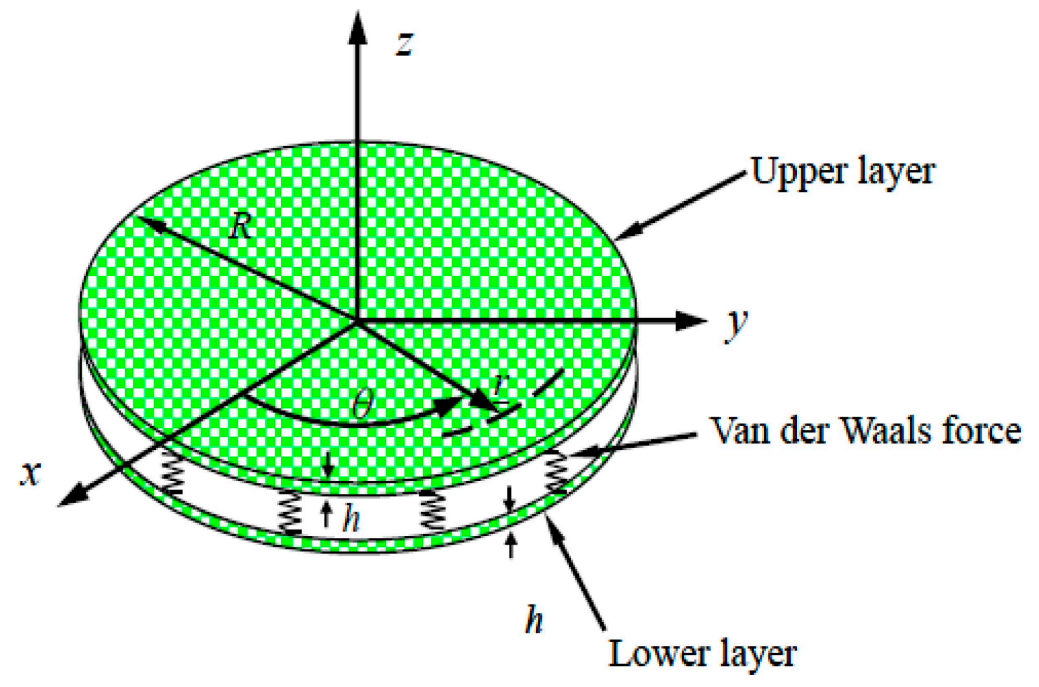

Fig. 1. Geometry of a clamped double-layer graphene sheets

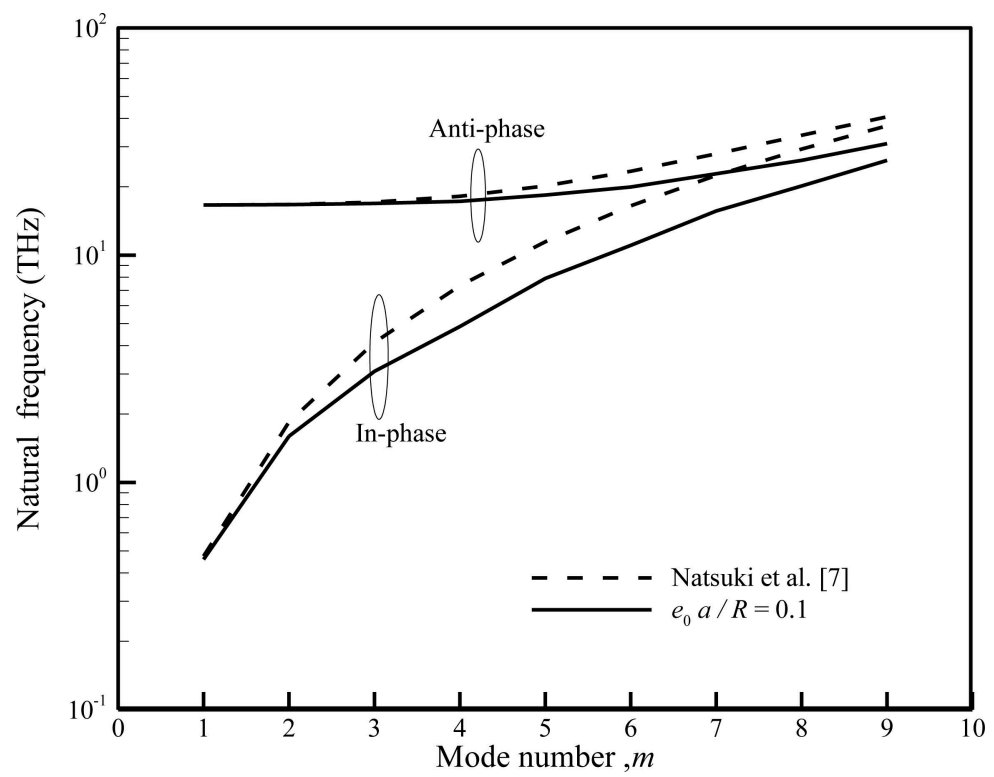

Fig. 2. The in-phase and anti-phase frequencies of the graphene sheets with $n=0$ for different mode numbers 